\title{
Hiperostosis frontal interna
}

\author{
Hyperostosis Frontalis Interna
}

\section{Elisa Ruiz-Tagle Fernández}

Médico Forense

\section{Resumen}

La Hiperostosis Frontal Interna (HFI) es una entidad caracterizada por un crecimiento óseo desmesurado a nivel de la tabla interna del hueso frontal. Clásicamente esta manifestación se ha visto incluida dentro de procesos muy distintos, muchos de ellos asociados a trastornos mentales, aunque en la actualidad se tiende a considerarla cada vez más un signo independiente (3). En este artículo se expone un caso de hiperostosis frontal interna fruto de un hallazgo casual durante la práctica de la autopsia judicial de una mujer de mediana edad con antecedentes de epilepsia y patología psiquiátrica.

\section{Palabras claves}

Hiperostosis Frontal Interna, Tabla interna, Hallazgo de autopsia.

\section{Summary}

Hyperostosis frontalis interna (HFI) is a disorder characterized by progressive bone accretion on the internal lamina of the frontal bone. Traditionally, this condition has been included in several syndromes, many of them related with psyquiatric diseases. However, recent publications consider HFI as an independient entity. This article reported a HFI casual finding during the autopsy of a medium age woman with neuropsychiatric symptons.

\section{Key words}

Hyperostosis frontalis interna, internal lamina, casual finding. 


\section{Introducción}

La HFI fue descrita por primera vez por Morgagni en el siglo XVII como parte de un cuadro que asociaba a su vez obesidad y virilismo. Desde entonces, tradicionalmente su aparición se ha tratado de relacionar con otros síndromes que incorporaban sintomatología de tipo psiquiátrico y endocrinológica preferentemente $(2,7)$. Sin embargo, estudios más actuales tienden a considerarla como una entidad independiente $(3,4,7,10)$.

Macroscópicamente la HFI se caracteriza por un crecimiento anormal de la tabla interna del hueso frontal a ambos lados de la línea media, a la que no llega a afectar $(3,10)$, mostrando así un aspecto que ha sido descrito por alguno autores como de «alas de mariposa» (11). Por otro lado, esta afectación se circunscribe a la parte endocraneal del hueso frontal, no suele afectar a la diploe o la porción externa del mismo (6), y rara vez se extiende más allá de los límites marcados por la sutura coronal o el recorrido de la arteria meníngea media $(3,9)$.

En su amplio estudio, Hershkowitz propuso una clasificación de hasta cuatro grados o niveles [A, B, C y D] según la extensión de esta lesión a lo largo del hueso frontal (3). Este ordenamiento ha sido mayoritariamente seguido por las publicaciones posteriores sobre el tema $(2,6,7)$, aunque en algunos casos se ha manifestado la dificultad para identificar los estadios más precoces (A y $B$ ), especialmente en radiografías anteroposteriores (2).

Aunque la prevalencia de la HFI en población actual varía, según la bibliografía consultada entre valores que rondan el 1\% (2) y aproximadamente $12 \%$ $(3,7,10)$, todos los estudios coinciden en que su aparición es notoriamente mayor en el sexo femenino, en una proporción de casi 9:1 (1), llegando a constituir un hallazgo relativamente frecuente en mujeres de edad media avanzada $(2,3,6,10)$. Entre la población masculina parece ser una manifestación poco frecuente y asociada a casos de atrofia testicular (7).

Sin embargo, al analizar material proveniente de contextos arqueológicos su incidencia disminuye considerablemente. La principal razón que se ha esgrimido es que la menor esperanza de vida propia de poblaciones antiguas actuaría como factor limitante en la aparición de este fenómeno (1). Hershkowitz no encontró ningún caso de HFI en el estudio que realizó sobre los restos de más de 2.000 individuos provenientes de poblaciones históricas (3). Sólo estudios puntuales, como el realizado sobre restos de nativos americanos provenientes de Pueblo Bonito, Nuevo Méjico, llegan a ofrecer frecuencias de aparición de HFI similares a las encontradas en poblaciones actuales (5).

En relación con su etiología, la mayor parte de los investigadores parecen estar de acuerdo en la existencia de un desequilibrio hormonal $(3,7,10)$. Una de las principales relaciona la aparición de la HFI con un estimulación estrogénica prolongada (10), hecho que reactivaría los centros de osificación situados en el hueso frontal (7). De hecho, uno de los pocos casos de HFI en un hombre fue descrito en un varón con diagnóstico de síndrome de Klinefelter (8), patología que cursa con niveles aumentados de estradiol. Otras sin embargo, se apoyan más en los cambios microevolutivos de carácter hormonal debidos a mejoría en el estado nutricional de los individuos (9), lo que explicaría a su vez la mayor incidencia de la HFI en poblaciones actuales. En cualquier caso ninguna teoría ha sido a día de hoy plenamente aceptada (7).

Su significado clínico tampoco parece estar aclarado. Aunque inicialmente se relacionó con cefaleas, epilepsia y cuadros de demencia principalmente, en la actualidad parece aceptarse que se trata de un fenómeno aislado, que en la mayoría de los casos no induce ningún tipo de síntomas (7) y que suele reve- 
larse como un hallazgo casual durante la práctica de alguna prueba de imagen.

\section{Un caso clínico}

Presentamos el caso de una mujer, de 54 años, que fallece de forma violenta tras precipitarse desde la ventana de su vivienda, situada en un segundo piso. La fallecida vivía sola desde hacía varios años. Sus familiares más próximos la definen como una persona de «trato difícil», con tendencia al aislamiento e importantes alteraciones conductuales y del estado de ánimo.

La revisión de la historia clínica mostró como antecedentes de interés hipercolesterolemia, neuropatía cubital bilateral con parestesias residuales, cefalea crónica tensional, tres intentos autolíticos por sobreingesta medicamentosa y cuadro de epilepsia de larga evolución, al parecer con mal control terapéutico.

En el examen externo del cadáver, el único aspecto destacable era una lesión contusa abierta a nivel occipital izquierdo. Tras la apertura de la bóveda craneal y la retirada del encéfalo se observó línea de fractura que se extendía desde el lado izquierdo de la fosa craneal posterior hasta el techo de la órbita izquierda.

Al examinar la calota craneal, sin embargo, si se evidenció un importante sobrecrecimiento óseo a nivel frontal, que se extendía en dos áreas de aspecto simétrico sin llegar a afectar la línea media del hueso. En ambos lados la extensión de la lesión era parecida (4'6 × 6'2 cm el lado izquierdo; 5'2 × 6'4 cm el lado derecho) y presentaba la misma imagen de forma algodonosa y coloración nacarada. El espesor del hueso mostraba también un engrosamiento similar $\left(1^{\prime} 6 \mathrm{~cm}\right)$.

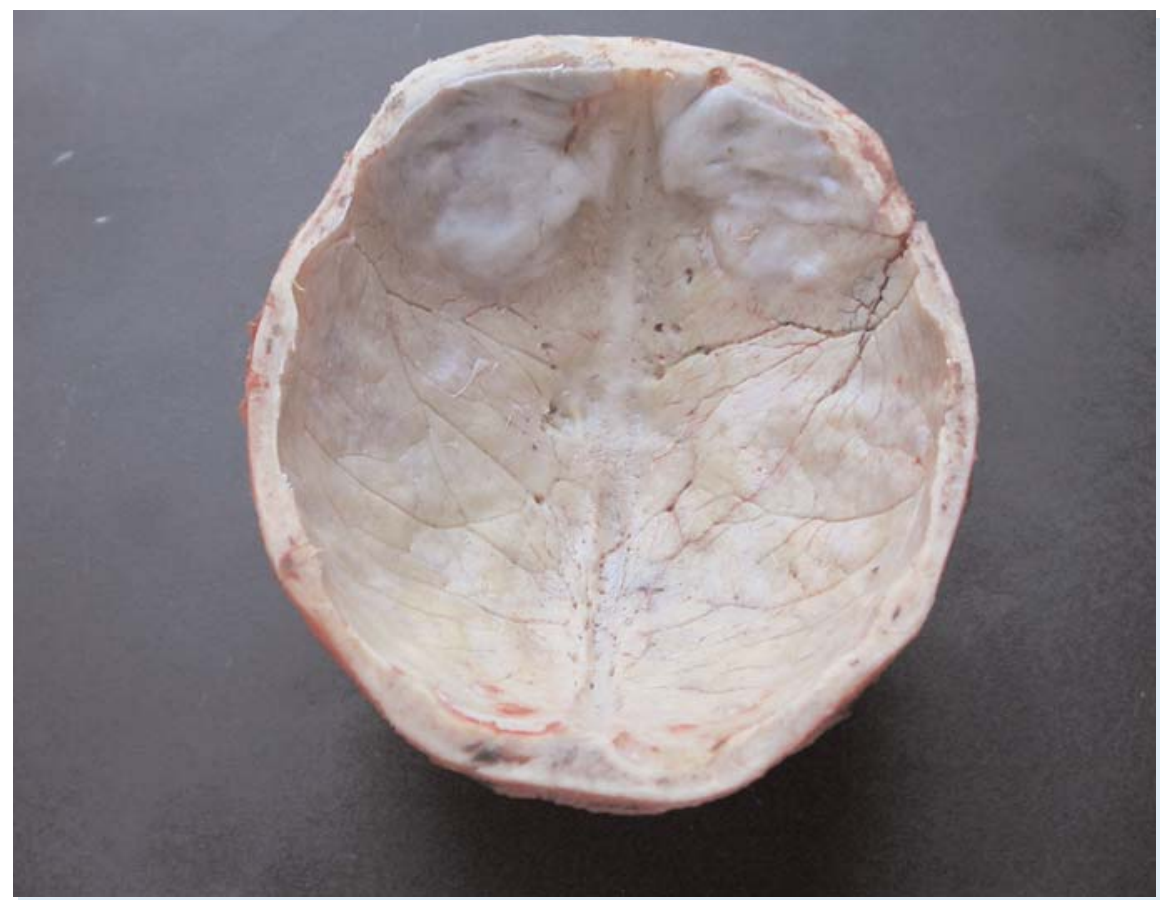

Figura 1. Imagen que muestra las dos zonas de sobrecrecimiento óseo a ambos lados de la línea media del hueso frontal 


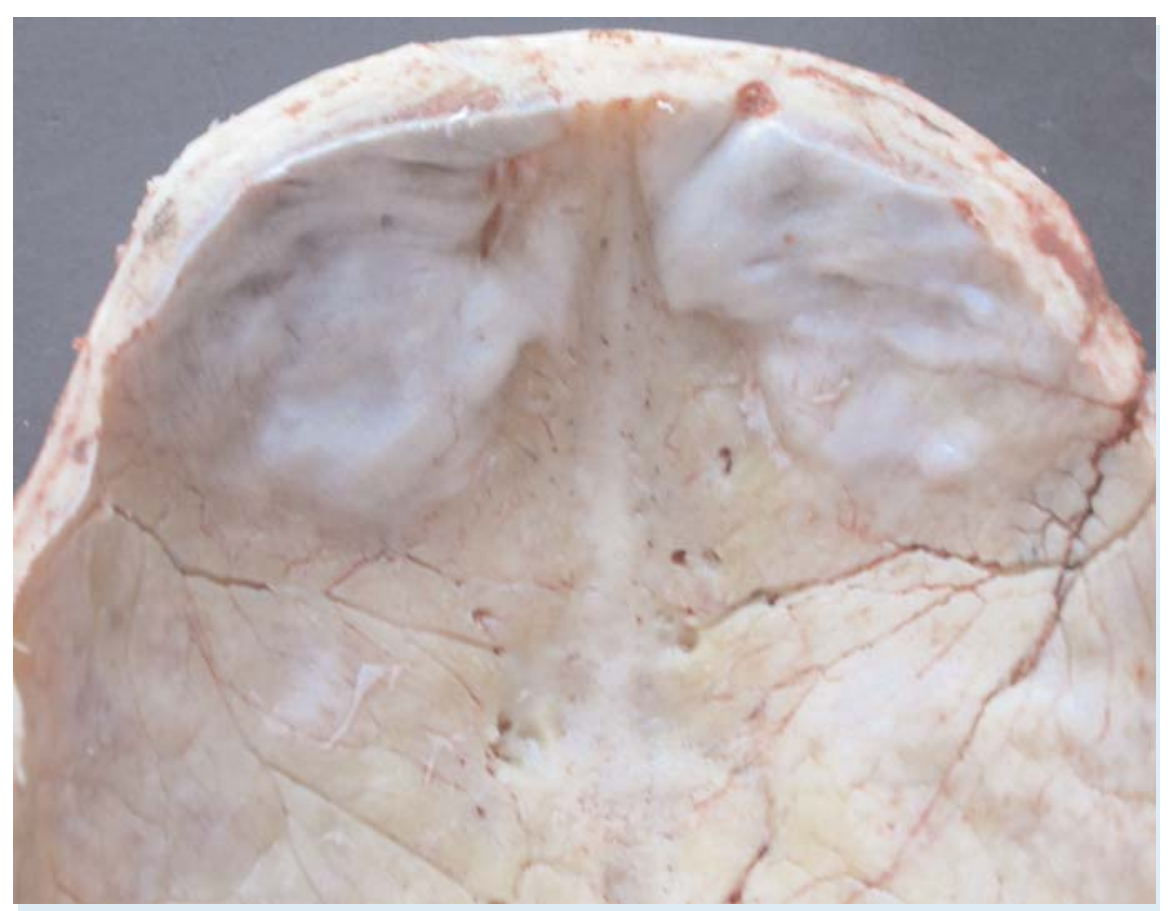

Figura 2. Ampliación de la imagen anterior.

Nótese la coloración nacarada del hueso de nueva formación

El análisis del resto de las estructuras craneales no mostró otras lesiones, salvo las relacionados con el mecanismo que produjo la muerte, ni tampoco se evidenció ningún tipo de daño a nivel encefálico que se pudieran relacionar con este descubrimiento.

\section{Conclusiones}

La HFI es un hallazgo casual durante la práctica de la autopsia. A día de hoy, las últimas investigaciones tienden a considerarla como una entidad propia, con mayor prevalencia entre las mujeres postmenopaúsicas y que no muestra una relación directa con ninguna patología determinada. En este sentido se valora el caso presentado.

\section{Bibliografía}

1 BARBer, G., WATT, I., Rogers, J. A comparison of radiological and paleopathological diagnostic criteria for hyperostosis frontalis interna. Int J Osteoarchaeol 1997; 7 (2); 157164.

2 Devriendt, W., Piercecchi-Marti, M.D., Adalian, P. et Al. Hyperostosis frontalis interna: forensic issues. J Forensic Sci 2005; 50 (1), 143-146.

3 Hershkovitz, I., Greenwald, C., Rothschild, B.M., et al. Hyperostosis frontalis interna: an anthropological perspective. Am J Phys Anthropol 1999; 109 (3), 303-325.

4 Kocabas H., Sezer, I., Melikoglu, M.A. et Al. Hyperostosis frontalis interna in a patient with giant cell arteritis. Mod Rheumatol 2008; 18, 181-183.

5 Mulhern, D.M., WilzAC, C.A., DudAR, J.C. Brief communication: unusual finding al Pueblo Bonito: multiple cases of hyperostosis frontalis interna. Am J Phys Anthropol 2006; 130 (4), 480-484.

6 Nikolic, S., DJONIC, D., ZIVKoVIC, V. ET AL. Rate of occurrence, gross appearance and age 
relation of hyperostosis frontalis interna in females: a prospective autopsy study. Am J Forensic Med Pathol 2010; 31 (3), 205-207.

7 Raikos, A., Paraskevas, G. K., Yusuf, F. et Al. Ethiopathogenesis of hyperostosis frontalis interna: a mistery skill. Ann Anat 2011; 193 (5), 453-458.

8 RamChANDREN S., LIEBESKIND D.S. Headache in a patient with Klinefelter's syndrome and hyperosotsis frontalis interna. J Headache Pain 2007; 8, 342-344.

9 RÜHLI, F.J., HenNenBerg, M. Are hyperostosis frontalis interna and leptin linked? A hypothetical approach about hormonal influence on human microevolution. Med Hypotheses 2002; 58 (5), 378-381.

10 SHE, R., SzAKacs, J. Hyperostosis frontalis interna: case report and review of literature. Ann Clin Lab Sci 2004; 34 (2), 206-208.

11 Talarico JR, E.F., Prather, A.D., Hardt, K.D. A case of extensive hyperostosis frontalis interna in an 87-years-old female human cadaver. Clin Anat 2008; 21 (3), 259-268. 